\title{
FINITELY ADDITIVE SUPERMARTINGALES
}

\author{
GIANLUCA CASSESE
}

\begin{abstract}
The concept of finitely additive supermartingales, originally due to Bochner, is revived and developed. We exploit it to study measure decompositions over filtered probability spaces and the properties of the associated Doléans-Dade measure. We obtain versions of the Doob Meyer decomposition and, as an application, we establish a version of the Bichteler and Dellacherie theorem with no exogenous probability measure.
\end{abstract}

\section{INTRODUCTION}

In the classical theory of probability one often encounters situations in which countable additivity fails. Broadly speaking, these fall into two main classes of problems: those involving duality on the space $L^{\infty}$ and those in which the underlying $\sigma$ algebra needs to be extended, e.g. to overcome the lack of measurability of some random quantity. Both situtations are well documented in applications. [18, is a recent example of the former kind of situations arising in mathematical finance. In the area of weak convergence it is well known that even the classical empirical process is not Borel measurable in the space $D[0,1]$ when the latter is equipped with the non separable topology induced by the supremum norm. Dudley [14 illustrates a number of situations relevant for empirical processes in which measurability fails. To overcome these drawbacks, a new approach, based on outer expectation, was developed by Hoffmann-Jorgensen and, in a systematic way, in the book by van der Vaart and Wellner [26. More recently Berti and Rigo [4 have shown that such notion of weak convergence has an exact translation in the language of finite additivity.

In this paper we fix an algebra $\mathcal{A}$ of subsets of some set $\Omega$ and an increasing family $\left(\mathcal{A}_{t}: t \in \mathbb{R}_{+}\right)$of sub algebras of $\mathcal{A}$, a filtration $\left(\mathcal{F}\right.$ and $\mathcal{F}_{t}$ will hereafter denote the $\sigma$ algebras generated by $\mathcal{A}$ and $\mathcal{A}_{t}$ respectively). To illustrate our topic, consider the quantity $m\left(f_{t}\right)$ where $f=\left(f_{t}: t \in \mathbb{R}_{+}\right)$is an adapted process and $m$ a finitely additive probability on $\mathcal{A}$, that is $m$ is a positive, finitely additive set function on $\mathcal{A}$ (in symbols $\left.m \in b a(\mathcal{A})_{+}\right)$and $m(\Omega)=1$. When dealing with finitely additive expectation, of special importance are the structural properties of $m$ such as decompositions, particularly the one of Yosida and Hewitt [27. In our setting, however, what matters are the properties of $m$ conditional on $\mathcal{A}_{t}$ and the focus then shifts from the finitely additive measure $m$ to the finitely additive process $\left(m_{t}: t \in \mathbb{R}_{+}\right)$where $m_{t}=m \mid \mathcal{A}_{t}$; or even $\left(m_{t}^{c}: t \in \mathbb{R}_{+}\right.$), where $m_{t}^{c}$ and $m_{t}^{\perp}$ designate the countably and purely finitely additive components of $m_{t}$ (in the sequel the spaces of countably and purely finitely additive set functions on an algebra $\mathcal{G}$ will be indicated with the symbols $c a(\mathcal{G})$ and $p f a(\mathcal{G})$ respectively while $\mathbb{P}(\mathcal{G})$ will be used to designate full probabilities, i.e. countably additive, on $\mathcal{G}$ ). The inclusion $\mathcal{A}_{t} \subset \mathcal{A}_{u}$ for $u \geq t$ implies $m_{u}^{c} \mid \mathcal{A}_{t} \leq m_{t}$ and $m_{t}^{\perp} \perp m_{u}^{c} \mid A_{t}$ i.e. $m_{u}^{c} \mid \mathcal{A}_{t}=m_{t} \wedge\left(m_{u}^{c} \mid \mathcal{A}_{t}\right) \leq m_{t}^{c}+\left(m_{t}^{\perp} \wedge\left(m_{u}^{c} \mid \mathcal{A}_{t}\right)\right)=m_{t}^{c}$, a conclusion which extends to any decomposition

Date: November 7, 2018

2000 Mathematics Subject Classification. Primary 28A12,60G07, 60G20.

Key words and phrases. Bichteler Dellacherie theorem, Conditional expectation, Doléans-Dade measure, Doob Meyer decomposition, finitely additive measures, supermartingales, Yosida Hewitt decomposition.

I am in debt with an anonymous referee for several helping suggestions. All remaining erros are my own. 
$m_{t}=m_{t}^{e}+m_{t}^{p}$, such that $m_{t}^{p} \perp m_{u}^{e} \mid \mathcal{A}_{t}$ - see e.g. Lemma 2. $\left(m_{t}: t \in \mathbb{R}_{+}\right)$and $\left(m_{t}^{c}: t \in \mathbb{R}_{+}\right)$turn thus out being finitely additive supermartingales, a concept introduced by Bochner, in a number of little known papers - [6], [7] and [8] - and later revived by Armstrong in [2] and [3].

More formally, a finitely additive stochastic process $\xi=\left(\xi_{t}: t \in \mathbb{R}_{+}\right)$is an element of the vector lattice $\prod_{t \in \mathbb{R}_{+}} b a\left(\mathcal{A}_{t}\right)$ endowed with the order induced by each coordinate space. $\xi$ is a finitely additive supermartingale if

$$
\xi_{t}(F) \geq \xi_{u}(F) \quad F \in \mathcal{A}_{t}, t \leq u
$$

The symbol $\mathfrak{S}$ designates the set of finitely additive supermartingales such that $\|\xi\| \equiv \sup _{t \in \mathbb{R}_{+}}\left\|\xi_{t}\right\|<\infty$. With no loss of generality we put $\mathcal{A}_{0}=\bigcap_{t \in \mathbb{R}_{+}} \mathcal{A}_{t}$ and $\mathcal{A}=\bigcup_{t \in \mathbb{R}_{+}} \mathcal{A}_{t}$ and define, for $F \in \mathcal{A}$ and $G \in \mathcal{A}_{0}$, $\xi_{\infty}(F)=\inf _{\left\{t: F \in \mathcal{A}_{t}\right\}} \xi_{t}(F)$ and $\xi_{0}(G)=\sup _{t} \xi_{t}(G)$ a choice that will allow us to replace $\mathbb{R}_{+}$by $\overline{\mathbb{R}}_{+}=$ $\mathbb{R}_{+} \cup\{\infty\}$ when necessary. We also use the symbols $\bar{\Omega}=\Omega \times \overline{\mathbb{R}}_{+}$and $\overline{\mathcal{F}} \equiv \mathcal{F} \otimes\left\{\varnothing, \mathbb{R}_{+}\right\}$.

We use repeatedly the following corollary of the Hahn-Banach theorem (see e.g. [5, 3.2.3(b) and 3.2.10]).

Lemma 1. If $\Sigma_{0} \subset \Sigma$ are algebras of subsets of some set $S$ and $\mu_{0} \in b a\left(\Sigma_{0}\right)_{+}$then there exists $\mu \in b a(\Sigma)_{+}$ such that $\mu \mid \Sigma_{0}=\mu_{0}$.

Although all processes in this paper are indexed by $\mathbb{R}_{+}$, we often do not use but the order properties of the real numbers so that some of the results that follow carry over almost unchanged to the case of a linearly ordered index set.

\section{Finitely Additive Conditional Expectation}

The absence of a satisfactory concept of conditional expectation in the finitely additive setting, a major argument in favour of countable additivity, is a direct consequence of the failure of the Radon Nikodym theorem. The operator defined hereafter, e.g., provides an extension of such fundamental concept which is suitable for many analytical purposes but lacks some of the properties which matter for the sake of its statistical interpretation (a different proof of the following result appears in [10, proposition 2.1, p. 27]).

Theorem 1. Let $\mathcal{H}$ be an algebra of subsets of $\Omega, \mathcal{G}$ a sub $\sigma$ algebra of $\mathcal{H}$, and $\mu \in b a(\mathcal{H})_{+}$. Let $\mu \mid \mathcal{G}$ decompose as $\lambda+\pi$, with $\lambda \in c a(\mathcal{G})_{+}, \pi \in b a(\mathcal{G})_{+}$and $\lambda \perp \pi$ and define

$$
\mathcal{I}_{\pi}=\{G \in \mathcal{G}: \pi(G)=0\}
$$

If $f \in L^{1}(\mu)$ there exists a unique $\mu\left(f \mid \mathcal{I}_{\pi}\right) \in L^{1}(\lambda)$ such that

$$
\mu\left(f \mathbf{1}_{I}\right)=\mu\left(\mu\left(f \mid \mathcal{I}_{\pi}\right) \mathbf{1}_{I}\right)=\lambda\left(\mu\left(f \mid \mathcal{I}_{\pi}\right) \mathbf{1}_{I}\right) \quad I \in \mathcal{I}_{\pi}
$$

and

$$
\mu\left(f \mathbf{1}_{G} \mid \mathcal{I}_{\pi}\right)=\mu\left(f \mid \mathcal{I}_{\pi}\right) \mathbf{1}_{G} \quad G \in \mathcal{G}
$$

$\mu\left(\cdot \mid \mathcal{I}_{\pi}\right): L^{1}(\mu) \rightarrow L^{1}(\lambda)$ is a positive, linear operator with $\left\|\mu\left(\cdot \mid \mathcal{I}_{\pi}\right)\right\|=1$.

Proof. Being closed with respect to finite unions, $\mathcal{I}_{\pi}$ is a directed set relatively to inclusion. Since $\lambda \perp \pi$ and $\mathcal{G}$ is a $\sigma$ algebra, for each $\epsilon>0$ there exists $I \in \mathcal{I}_{\pi}$ such that $\lambda\left(I^{c}\right) \leq \epsilon$ : i.e. $\lambda(G)=\lim _{I \in \mathcal{I}_{\pi}} \lambda(I G), G \in \mathcal{G}$. Let $f \in L^{1}(\mu)$. Any solution $p(f) \in L^{1}(\lambda)$ to (2.2) must then satisfy

$$
\lambda\left(p(f) \mathbf{1}_{G}\right)=\lim _{I \in \mathcal{I}_{\pi}} \lambda\left(p(f) \mathbf{1}_{G \cap I}\right)=\lim _{I \in \mathcal{I}_{\pi}} \mu\left(f \mathbf{1}_{G \cap I}\right) \quad G \in \mathcal{G}
$$


and is therefore unique $P$ a.s.: by considering $f^{+}$and $f^{-}$separately we can (and will) thus restrict to the case in which $f \in L^{1}(\mu)_{+}$.

Let $\mu_{f} \in b a(\mathcal{G})_{+}$be defined implicitly by letting

$$
\mu_{f}(G)=\lim _{I \in \mathcal{I}_{\pi}} \mu\left(f \mathbf{1}_{G \cap I}\right) \quad G \in \mathcal{G}
$$

The limit in (2.4) exists uniformly with respect to $G \in \mathcal{G}$. In fact, $I \in \mathcal{I}_{\pi}$ implies $\mu_{f}(I)=\mu\left(f \mathbf{1}_{I}\right)$ and $\lim _{I \in \mathcal{I}_{\pi}} \mu_{f}\left(I^{c}\right)=0$ so that

$$
0 \leq \mu_{f}(G)-\mu\left(f \mathbf{1}_{G \cap I}\right)=\mu_{f}(G)-\mu_{f}(G \cap I) \leq \mu_{f}\left(I^{c}\right)
$$

Let $\left\langle G_{n}\right\rangle_{n \in \mathbb{N}} \subset \mathcal{G}$ be such that $\lim _{n} \lambda\left(G_{n}\right)=0$ and $I \in \mathcal{I}_{\pi}$. Then $\lim _{n} \mu\left(G_{n} \cap I\right)=\lim _{n} \lambda\left(G_{n} \cap I\right)=0$ so that $\lim _{n} \mu\left(f \mathbf{1}_{G_{n} \cap I}\right)=0$, by absolute continuity of the finitely additive integral [15, III.2.20(b)]. Then [15, I.7.6]

$$
\lim _{n} \mu_{f}\left(G_{n}\right)=\lim _{n} \lim _{I \in \mathcal{I}_{\pi}} \mu\left(f \mathbf{1}_{G_{n} \cap I}\right)=\lim _{I \in \mathcal{I}_{\pi}} \lim _{n} \mu\left(f \mathbf{1}_{G_{n} \cap I}\right)=0
$$

i.e. $\mu_{f} \ll \lambda$. (2.2) follows by letting $\mu\left(f \mid \mathcal{I}_{\pi}\right) \in L^{1}(\lambda)_{+}$be the corresponding Radon Nikodym derivative; (2.3) from $I G \in \mathcal{I}_{\pi}$ whenever $I \in \mathcal{I}_{\pi}$ and $G \in \mathcal{G} . \mu\left(\cdot \mid \mathcal{I}_{\pi}\right)$ is linear and positive as $\mu$ is. If $f \in L^{1}(\mu)$,

$$
\lambda\left(\left|\mu\left(f \mid \mathcal{I}_{\pi}\right)\right|\right) \leq \lim _{I \in \mathcal{I}_{\pi}} \lambda\left(\mu\left(|f| \mid \mathcal{I}_{\pi}\right) \mathbf{1}_{I}\right)=\lim _{I \in \mathcal{I}_{\pi}} \mu\left(|f| \mathbf{1}_{I}\right) \leq\|f\|
$$

with equality if $f$ is the indicator of some $I \in \mathcal{I}_{\pi}$ i.e. $\left\|\mu\left(\cdot \mid \mathcal{I}_{\pi}\right)\right\|=1$.

Referring to $\mu\left(\cdot \mid \mathcal{I}_{\pi}\right)$ as "conditional expectation" is just a convenient abuse of terminology as the law of total probability $\mu(f)=\mu\left(\mu\left(f \mid \mathcal{I}_{\pi}\right)\right)$, which is at the basis of the statistical interpretation of this concept [17, p. 1229], will in general not hold unless $\mu \mid \mathcal{G} \in c a(\mathcal{G}) 1$. Of course, if $\mu \in c a(\mathcal{H})$ the above concept of conditional expectation would coincide (by uniqueness) with the traditional one.

\section{The Doléans-Dade Measure}

In the early works of Doléans-Dade [12, Föllmer [16] and Metivier and Pellaumail [21, supermartingales were associated with measures over predictable rectangles. We address this issue in the present setting. The claims and the proofs of this this section remain true if we replace $\mathbb{R}_{+}$by any linearly ordered index set.

Denote by $\mathcal{R}$ the collection of all sets of the form

$$
\left.F_{0} \times\{0\} \cup \bigcup_{n=1}^{N} F_{n} \times\right] t_{n}, \infty[
$$

where $F_{0} \in \mathcal{A}_{0}, N \in \mathbb{N}$ and, for each $N \geq n>m \geq 1, F_{n} \in \mathcal{A}_{t_{n}}$ and $F_{n} \cap F_{m}=\varnothing$. $\mathcal{R}$ is closed with respect to intersection and contains $\bar{\Omega}$ and $\varnothing$. We denote by $\mathcal{P}$ the algebra generated by $\mathcal{R}$ : each $F \in \mathcal{P}$ takes then the form of a disjoint union

$$
\left.\left.F_{0} \times\{0\} \cup \bigcup_{n=1}^{N} F_{n} \times\right] t_{n}, u_{n}\right]
$$

with $F_{0} \in \mathcal{A}_{0}, t_{n}, u_{n} \in \mathbb{R}_{+}, F_{n} \in \mathcal{A}_{t_{n}}$. We also denote by $\overline{\mathcal{P}}$ the collection defined as in (3.2) but with $\mathcal{A}_{t}$ replaced by $\mathcal{A}$ for each $t \in \mathbb{R}_{+}$. Let

$$
\mathfrak{M}=\left\{\bar{x} \in b a\left(2^{\bar{\Omega}}\right)_{+}: \lim _{t} \bar{x}(\Omega \times] t, \infty[)=0\right\}
$$

\footnotetext{
${ }^{1}$ The failure of this property for reasonable definitions of finitely additive conditional expectation is well known since the work of Dubins 13
} 
Theorem 2. $\xi \in \mathfrak{S}$ if an only if there exists $\bar{x} \in \mathfrak{M}$ and $\lambda \in b a\left(\mathcal{A}_{\infty}\right)$ such that

$$
\xi_{t}(F)=\lambda(F)+\bar{x}(F \times] t, \infty[) \quad t \in \mathbb{R}_{+}, F \in \mathcal{A}_{t}
$$

Proof. Assume that $\xi \in \mathfrak{S}$ and, replacing $\xi_{t}$ with $\xi_{t}-\xi_{\infty}$, assume also that $\xi_{\infty}=0$. For each $F_{0} \times\{0\} \cup$ $\left.\bigcup_{n=1}^{N} F_{n} \times\right] t_{n}, \infty[\in \mathcal{R}$ define the quantity

$$
x\left(F_{0} \times\{0\} \cup \bigcup_{n=1}^{N} F_{n} \times\right] t_{n}, \infty[)=\sum_{n=1}^{N} \xi_{t_{n}}\left(F_{n}\right)
$$

Let $\left.F=F_{0} \times\{0\} \cup \bigcup_{n=1}^{N} F_{n} \times\right] t_{n}, \infty\left[\right.$ and $\left.G=G_{0} \times\{0\} \cup \bigcup_{k=1}^{K} G_{k} \times\right] u_{k}, \infty[$ be sets in $\mathcal{R}$. To prove that $\mathcal{R}$ is a lattice, write $F_{0}^{\prime}=F_{0} \cap G_{0}^{c}, G_{0}^{\prime}=G_{0}$ and, for $n, k>0, F_{n}^{\prime}=F_{n} \cap \bigcap_{\left\{k>0: u_{k} \leq t_{n}\right\}} G_{k}^{c}$ and $G_{k}^{\prime}=G_{k} \cap \bigcap_{\left\{n>0: t_{n}<u_{k}\right\}} F_{n}^{c}$. We obtain

$$
F \cup G=\left(F_{0}^{\prime} \cup G_{0}^{\prime}\right) \times\{0\} \cup\left(\bigcup_{n=1}^{N} F_{n}^{\prime} \times\right] t_{n}, \infty[) \cup\left(\bigcup_{k=1}^{K} G_{k}^{\prime} \times\right] u_{k}, \infty[) \in \mathcal{R}
$$

Rearrange the collection $\left\{t_{n}, u_{k}: 1 \leq n \leq N, 1 \leq k \leq K\right\}$ as $\left\langle\gamma_{i}\right\rangle_{i=1}^{I}$ with $\gamma_{i} \geq \gamma_{i+1}$ and set conventionally $\xi_{\gamma_{0}}=0$. For $1 \leq i \leq I$, let $\hat{\psi}_{i}$ be a positive extension of $\left(\xi_{\gamma_{i}}-\xi_{\gamma_{i-1}}\right) \mid \mathcal{A}_{\gamma_{i}}$ to $\mathcal{A}$ and $\hat{\xi}_{\gamma_{i}}=\sum_{j=1}^{i} \hat{\psi}_{j}$. Then, $\hat{\xi}_{\gamma_{i}} \mid \mathcal{A}_{\gamma_{i}}=\xi_{\gamma_{i}}$ and $\hat{\xi}_{\gamma_{i+1}} \geq \hat{\xi}_{\gamma_{i}} \geq 0$, i.e. $\hat{\xi}_{t_{n}} \geq \hat{\xi}_{u_{k}}$ whenever $t_{n} \leq u_{k}$. For $\left.\left.0<k \leq K, \bigcup_{n=1}^{N}\left(G_{k} \cap F_{n}\right) \times\right] u_{k}, t_{n}\right] \subset$ $F^{c} \cap G$ (as $F_{n} \cap F_{m}=\varnothing$ for $n>m>0$ ). Then $G \subset F$ implies $G_{k} \cap F_{n}=\varnothing$ for all $1 \leq k \leq K$ and $1 \leq n \leq N$ such that $u_{k}<t_{n}$ that is $G_{k}=\bigcup_{\left\{n: t_{n} \leq u_{k}\right\}}\left(G_{k} \cap F_{n}\right)$. Therefore,

$$
\sum_{k=1}^{K} \xi_{u_{k}}\left(G_{k}\right)=\sum_{\left\{1 \leq n \leq N, 1 \leq k \leq K: t_{n} \leq u_{k}\right\}} \xi_{u_{k}}\left(G_{k} \cap F_{n}\right) \leq \sum_{\left\{1 \leq n \leq N, 1 \leq k \leq K: t_{n} \leq u_{k}\right\}} \hat{\xi}_{t_{n}}\left(G_{k} \cap F_{n}\right) \leq \sum_{n=1}^{N} \xi_{t_{n}}\left(F_{n}\right)
$$

The set function $x$ defined in (3.5) is then monotonic and a fortiori well defined. If $F=\varnothing$ then $\bigcup_{n=1}^{N} F_{n}=\varnothing$ so that $x(F)=0$; moreover

$$
\begin{aligned}
x(F \cup G)= & \sum_{n=1}^{N} \xi_{t_{n}}\left(F_{n}^{\prime}\right)+\sum_{k=1}^{K} \xi_{u_{k}}\left(G_{k}^{\prime}\right) \\
= & \sum_{n=1}^{N} \xi_{t_{n}}\left(F_{n}\right)-\sum_{\left\{1 \leq n \leq N, 1 \leq k \leq K: t_{n} \geq u_{k}\right\}} \xi_{t_{n}}\left(F_{n} \cap G_{k}\right) \\
& +\sum_{k=1}^{K} \xi_{u_{k}}\left(G_{k}\right)-\sum_{\left\{1 \leq n \leq N, 1 \leq k \leq K: t_{n}<u_{k}\right\}} \xi_{u_{k}}\left(F_{n} \cap G_{k}\right) \\
= & \sum_{n=1}^{N} \xi_{t_{n}}\left(F_{n}\right)+\sum_{k=1}^{K} \xi_{u_{k}}\left(G_{k}\right)-\sum_{k, n \geq 1} \xi_{t_{n} \vee u_{k}}\left(F_{n} \cap G_{k}\right) \\
= & x(F)+x(G)-x(F \cap G)
\end{aligned}
$$

In other words, $x$ is a strongly additive set function on a lattice of sets which contains $\varnothing$ as well as $\bar{\Omega}$ : as such [5, 3.1.6, 3.2.1 and 3.2.5] it admits an extension $\hat{x}$ to $\mathcal{P}$. If $F \in \mathcal{P}$ is as in (3.2), then

$$
\hat{x}(F)=\sum_{n=1}^{N}\left\{x\left(F_{n} \times\right] t_{n}, \infty[)-x\left(F_{n} \times\right] u_{n} \vee t_{n}, \infty[)\right\}
$$

$\hat{x}$ is thus unique and, in view of (3.6), positive. But then, $\hat{x}$ admits a positive extension $\bar{x}$ to $2^{\bar{\Omega}}$, by Lemma 1. If $\bar{x} \in \mathfrak{M}$ and $\lambda \in b a\left(\mathcal{A}_{\infty}\right)$ are given and $\xi$ is defined as in (3.4) then it is clear that $\xi \in \mathfrak{S}$ with $\xi_{\infty}=\lambda$ 
If $\xi \in \mathfrak{S}$, then denote by $\mathfrak{M}(\xi)$ the collections of those $\bar{x} \in \mathfrak{M}$ meeting (3.4). Each $\bar{x} \in \mathfrak{M}(\xi)$ will be referred to as a Doléans-Dade measure associated to $\xi$. Disregarding the apparent arbitrariness implicit in the existence of a multiplicity of such measures, there are several remarkably simple implications of Theorem 2 on such relevant issues as the decomposition and extension of supermartingales that are spelled out in the next corollaries where the following notation is used: let $\mathfrak{M}^{u c}, \mathfrak{M}^{u p} \subset \mathfrak{M}$ consist of measures $m$ such that $m \mid \overline{\mathcal{F}} \in \operatorname{ca}(\overline{\mathcal{F}})$ and $m \mid \overline{\mathcal{F}} \in \operatorname{pfa}(\overline{\mathcal{F}})$, respectively. We set

$$
\mathfrak{S}^{u c}=\left\{\xi \in \mathfrak{S}: \xi_{\infty} \in c a(\mathcal{A}), \mathfrak{M}(\xi) \cap \mathfrak{M}^{u c} \neq \varnothing\right\} \quad \text { and } \quad \mathfrak{S}^{u p}=\left\{\xi \in \mathfrak{S}: \xi_{\infty} \in p f a(\mathcal{A}), \subset \mathfrak{M}^{u p}\right\}
$$

A supermartingale belonging to $\mathfrak{S}^{u c}$ (resp. $\mathfrak{S}^{u p}$ ) will be called uniformly countably additive (resp. uniformly purely finitely additive).

Corollary 1. Any $\xi \in \mathfrak{S}$ admits a decomposition

$$
\xi=\mu-\alpha
$$

where $\mu$ is a finitely additive martingale and $\alpha$ a positive, finitely additive increasing process (as defined in [2, p. 287]). Moreover, the following are equivalent:

(i) $\xi \in \mathfrak{S}^{u c}$

(ii) $\mu$ and $\alpha$ in (3.8) may be chosen such that $\mu_{\infty}, \alpha_{\infty} \in c a(\mathcal{A})$ (and thus so that $\mu$ and $\alpha$ are countably additive processes);

(iii) there exists $\lambda \in c a(\mathcal{A})_{+}$such that $\left|\xi_{t}\right| \leq \lambda \mid \mathcal{A}_{t}$ for each $t \in \mathbb{R}_{+}$.

Proof. Let $\bar{x} \in \mathfrak{M}(\xi)$ and define

$$
\mu_{t}(F)=\xi_{\infty}(F)+\bar{x}\left(F \times \mathbb{R}_{+}\right) \quad \text { and } \quad \alpha_{t}(F)=\bar{x}(F \times[0, t]) \quad F \in \mathcal{A}_{t}
$$

Then (3.8) follows from (3.4). In fact $\mu$ is a finitely additive martingale while $\alpha$ extends to an increasing family $\left(\bar{\alpha}_{t}: t \in \mathbb{R}_{+}\right)$of measures on $\mathcal{A}$ such that $\inf _{t}\left\|\bar{\alpha}_{t}\right\|=\left\|\alpha_{0}\right\|=0$. If $(i)$ holds, then upon choosing $\bar{x} \in \mathfrak{M}(\xi)$ such that $\bar{x} \mid \overline{\mathcal{F}}$, 3.9.9) implies $(i i)$. If $(i i)$ holds let $\lambda=\left|\mu_{\infty}\right|+2 \alpha_{\infty}$. Then in restriction to $\mathcal{A}_{t}$ we obtain $\left|\xi_{t}\right| \leq\left|\xi_{\infty}\right|+\left(\xi_{t}-\xi_{\infty}\right) \leq\left|\mu_{\infty}\right|+\alpha_{\infty}+\left(\alpha_{\infty}-\alpha_{t}\right) \leq \lambda$ and (iii) follows. Assume $(i i i)$, then $\xi_{\infty} \in c a(\mathcal{A})$. Let $U=\left\{0=t_{1} \leq \ldots \leq t_{N}\right\}$ and define $\bar{\zeta}_{t_{N}}^{U} \in c a(\mathcal{A})_{+}$to be an extension of $\xi_{t_{N}}-\xi_{\infty} \mid \mathcal{A}_{t_{N}}$ to $\mathcal{A}$ dominated by $\lambda-\xi_{\infty}$ and set $\bar{\xi}_{t_{N}}^{U}=\xi_{\infty}+\zeta_{t_{N}}^{U}$; likewise, for $n<N$ let $\bar{\zeta}_{t_{n}}^{U} \in c a(\mathcal{A})_{+}$be an extension of $\xi_{t_{n}}-\xi_{t_{n+1}} \mid \mathcal{A}_{t_{n}}$ to

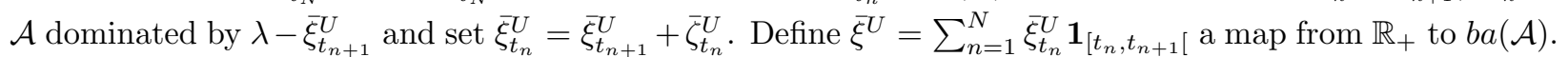
One easily establishes that $\xi_{\infty} \leq \bar{\xi}_{t}^{U} \leq \lambda$ for each $t \in \mathbb{R}_{+}$, i.e. $\bar{\xi}^{U} \in\left[\xi_{\infty}, \lambda\right]^{\mathbb{R}_{+}}$, that $\bar{\xi}^{U}$ is decreasing and that $\bar{\xi}_{t}^{U} \mid \mathcal{A}_{t}=\xi_{t}$ when $t \in U$. If $b a(\mathcal{A})^{\mathbb{R}_{+}}$is equipped with the product topology obtained after endowing each coordinate space with the weak* topology, we conclude that $\left[\xi_{\infty}, \lambda\right]^{\mathbb{R}_{+}}$is compact and that the net $\left\langle\bar{\xi}^{U}\right\rangle_{U \in \mathcal{U}}$, with $\mathcal{U}$ denoting the collection of finite subsets of $\mathbb{R}_{+}$directed by inclusion, admits a cluster point $\bar{\xi}$. Then necessarily, $\bar{\xi}$ is decreasing and $\bar{\xi}_{t} \mid \mathcal{A}_{t}=\xi_{t}$ for each $t \in \mathbb{R}_{+}$. The same argument used in the proof of Theorem 2 shows that the quantity $\sum_{n=1}^{N}\left(\bar{\xi}_{t_{n}}-\bar{\xi}_{u_{n}}\right)\left(F_{n}\right)$, where $\left.\left.F_{0} \times\{0\} \cup \bigcup_{n=1}^{N} F_{n} \times\right] t_{n}, u_{n}\right] \in \overline{\mathcal{P}}$, implicitly defines a measure on $\overline{\mathcal{P}}$ which admits an extension $\bar{x} \in \mathfrak{M}(\xi)$ such that $\bar{x}\left(F \times \mathbb{R}_{+}\right)=\left(\bar{\xi}_{0}-\bar{\xi}_{\infty}\right)(F) \leq \lambda(F)$ so that $\bar{x} \mid \overline{\mathcal{F}} \in \operatorname{ca}(\overline{\mathcal{F}})$.

Corollary 1 establishes a general version of the Doob Meyer decomposition. In addition it characterises exactly those processes $\xi$ admitting a countably additive version of such decomposition. This characterisation implies a condition hinging on the uniform countable additivity of the process $\xi$ or, equivalently, a weak form 
of countable additivity of the Doléans-Dade measure, namely $\bar{x} \mid \overline{\mathcal{F}} \in c a(\overline{\mathcal{F}})$. We shall return on this issue in the following sections.

The existence of Doléans-Dade measures easily translates into that of extensions of finitely additive supermartingales, a result which may prove useful in problems involving changes of the underlying filtration. For $H \subset \bar{\Omega}$ let $H_{\omega}$ denote the section $\left\{t \in \mathbb{R}_{+}:(\omega, t) \in H\right\}$.

Corollary 2. Consider an increasing family $\left(\mathcal{A}_{\tau}: \tau \in \mathbf{T}\right)$ of algebras of subsets of $\Omega$ where $\mathbf{T} \subset 2^{\bar{\Omega}}$ is ordered by reverse inclusion and let $\xi \in \mathfrak{S}$ and $\bar{x} \in \mathfrak{M}(\xi)$. There exists a finitely additive supermartingale $\xi^{*}$ on $\left(\mathcal{A}_{\tau}: \tau \in \mathbf{T}\right)$ such that $\bar{x} \in \mathfrak{M}\left(\xi^{*}\right)$. As a consequence

(i) If $\tau(t) \equiv \Omega \times] t, \infty\left[\in \mathbf{T}\right.$ and $F \in \mathcal{A}_{t} \cap \mathcal{A}_{\tau(t)}$ then $\xi_{\tau(t)}^{*}(F)=\xi_{t}(F)$

(ii) If $\tau, v \in \mathbf{T}, F \subset \Omega$ and $F_{\tau, v} \equiv\left\{\omega \in F: v_{\omega} \subset \tau_{\omega}\right\} \in \mathcal{A}_{\tau} \cap \mathcal{A}_{v}$ then $\xi_{\tau}^{*}\left(F_{\tau, v}\right) \geq \xi_{v}^{*}\left(F_{\tau, v}\right)$

Proof. Fix $\bar{x} \in \mathfrak{M}(\xi)$ and define $\xi_{\tau}^{*} \in b a\left(\mathcal{A}_{\tau}\right)$ implicitly by letting

$$
\xi_{\tau}^{*}(F)=\bar{\xi}_{\infty}(F)+\bar{x}\left(\left(F \times \overline{\mathbb{R}}_{+}\right) \cap \tau\right) \quad F \in \mathcal{A}_{\tau}
$$

where $\bar{\xi}_{\infty}$ is an extension of $\xi_{\infty}$ to $2^{\Omega}$ : (i) is immediate from (3.5). Given that $\tau \leq v$ is equivalent to $v \subset \tau$ then $F \in \mathcal{A}_{\tau}$ and $\tau \leq v$ imply $\xi_{\tau}^{*}(F) \geq \xi_{v}^{*}(F)$ so that $\xi^{*}$ is a finitely additive supermartingale on $\left(\mathcal{A}_{\tau}: \tau \in \mathbf{T}\right)$. Moreover, if $F \subset \Omega$ and $F_{\tau, v} \in \mathcal{A}_{\tau} \cap \mathcal{A}_{v}$ then $\xi_{\tau}^{*}\left(F_{\tau, v}\right) \geq \xi_{v}^{*}\left(F_{\tau, v}\right)$ is equivalent to $\bar{x}\left(\left(F_{\tau, v} \times \overline{\mathbb{R}}_{+}\right) \cap \tau\right) \geq \bar{x}\left(\left(F_{\tau, v} \times \overline{\mathbb{R}}_{+}\right) \cap v\right)$ which follows from $\bar{x}$ being positive.

Whenever $\tau(t) \in \mathbf{T}$ and $\mathcal{A}_{t} \subset \mathcal{A}_{\tau(t)}$ for all $t \in \mathbb{R}_{+}$, Corollary 2 suggests that any $\xi \in \mathfrak{S}$ may be consistently extended to any filtration indexed by $\mathbf{T}$. Corollary 2 is an illustration of the importance of finite versus countable additivity.

\section{Two Decompositions}

We shal prove in this section that all finitely additive supermartingales have a component that may be represented as a classical supermartingale with respect to some $P \in \mathbb{P}(\mathcal{F})$. It should be highlighted that the probability measure $P$ involved here emerges endogenously, rather than being given from the outset, as in the classical theory. We start with a preliminary result.

Lemma 2. Let $\mathcal{G} \subset \mathcal{H}$ be two algebras of subsets of $\Omega$ and denote by $c a(\mathcal{G}, \mathcal{H})$ and pfa $(\mathcal{G}, \mathcal{H})$ the subspaces of $b a(\mathcal{G})$ consisting of set functions which admit a countably additive extension to $\mathcal{H}$ and whose norm preserving extensions to $\mathcal{H}$ are all purely finitely additive, respectively. For each $\lambda \in b a(\mathcal{G})$ there exists a unique way of writing

$$
\lambda=\lambda^{e}+\lambda^{p}
$$

where $\lambda^{e} \in c a(\mathcal{G}, \mathcal{H}), \lambda^{p} \in p f a(\mathcal{G}, \mathcal{H})$ and $\lambda^{e}, \lambda^{p} \geq 0$ if and only if $\lambda \geq 0$.

Proof. With the aid of the Radon Nikodym theorem it is easily seen that $\lambda \in c a(\mathcal{G}, \mathcal{H})$ if and only if $\lambda \ll \bar{\lambda} \mid \mathcal{G}$ for some $\bar{\lambda} \in c a(\sigma \mathcal{H})$ and, thus, that $c a(\mathcal{G}, \mathcal{H})$ is an ideal. Let $\left\langle\lambda_{\alpha}\right\rangle_{\alpha \in A}$ be an increasing net in $c a(\mathcal{G}, \mathcal{H})_{+}$ with $\bigvee_{\alpha \in \mathbf{A}} \lambda_{\alpha}=\lambda \in b a(\mathcal{G})$. Fix $\alpha_{1} \in \mathbf{A}$ arbitrarily and, for given $\alpha_{n-1}$, let $\alpha_{n} \geq \alpha_{n-1}$ be such that $\lambda_{\alpha_{n}}(\Omega) \geq \lambda(\Omega)-2^{-n}$. If $F \in \mathcal{G}$,

$$
\lambda(F) \geq \lim _{n} \lambda_{\alpha_{n}}(F)=\lambda(\Omega)-\lim _{n} \lambda_{\alpha_{n}}\left(F^{c}\right) \geq \lambda(\Omega)-\pi\left(F^{c}\right)=\lambda(F)
$$


But then, $\lambda \ll \sum_{n} 2^{-n} \lambda_{\alpha_{n}} \in c a(\mathcal{G}, \mathcal{H})$ i.e. $\lambda \in c a(\mathcal{G}, \mathcal{H})$. We obtain from Riesz theorem the decomposition $b a(\mathcal{G})=c a(\mathcal{G}, \mathcal{H})+c a(\mathcal{G}, \mathcal{H})^{\perp}$. The inclusion $p f a(\mathcal{G}, \mathcal{H}) \subset c a(\mathcal{G}, \mathcal{H})^{\perp}$ is clear. To prove the converse, let $\bar{\lambda} \in b a(\mathcal{H})$ extend $\lambda \in c a(\mathcal{G}, \mathcal{H})^{\perp}$. Then there exists $G_{n} \in \mathcal{G}$ such that $|\lambda|\left(G_{n}^{c}\right)+\left|\bar{\lambda}^{c}\right|\left(G_{n}\right)<2^{-n}$. If $G \in \mathcal{G}$

$$
|\lambda(G)|=\lim _{n}\left|\lambda\left(G \cap G_{n}\right)\right|=\lim _{n}\left|\bar{\lambda}^{\perp}\left(G \cap G_{n}\right)\right| \leq\left|\bar{\lambda}^{\perp}\right|(G)
$$

i.e. $\left\|\bar{\lambda}^{c}\right\|+\left\|\bar{\lambda}^{\perp}\right\|=\|\bar{\lambda}\|=\|\lambda\| \leq\left\|\bar{\lambda}^{\perp}\right\|$. In other words, $\lambda \in \operatorname{pfa}(\mathcal{G}, \mathcal{H})$.

Lemma 2 is a slight generalization of the classical decomposition of Yosida and Hewitt (by uniqueness the two decompositions coincide for $\mathcal{G}=\mathcal{H}$ ). It has though an important implication here as it implicitly suggests that finitely additive supermartingales may admit a component that can be represented as a classical supermartingale with respect to some $P \in \mathbb{P}(\mathcal{F})$.

Proposition 1. Let $\xi \in \mathfrak{S}_{+}$. For each $t \in \mathbb{R}_{+}$let $\xi_{t}=\xi_{t}^{e}+\xi_{t}^{p}$ with $\xi_{t}^{e} \in \operatorname{ca}\left(\mathcal{A}_{t}, \mathcal{F}\right)$ and $\xi_{t}^{p} \in \operatorname{pfa}\left(\mathcal{A}_{t}, \mathcal{F}\right)$ and set $\xi^{e}=\left(\xi_{t}^{e}: t \in \mathbb{R}_{+}\right)$and $\xi^{p}=\left(\xi_{t}^{p}: t \in \mathbb{R}_{+}\right)$. Then

$$
\xi=\xi^{e}+\xi^{p}
$$

is the unique decomposition of $\xi$ such that $\xi^{e} \in \mathfrak{S}_{+}$may be represented as a classical $P$ supermartingale $X$ for some $P \in \mathbb{P}(\mathcal{F}) 2$ while $\xi^{p}$ is positive and orthogonal to all finitely additive processes admitting such representation. We say that $\xi^{e}$ is representable and that the pair $(P, X)$ is a representation of $\xi^{e}$.

Proof. The inclusion $\xi^{e} \in \mathfrak{S}_{+}$was shown in the Introduction. As $\xi^{p}$ is clearly orthogonal to any classical stochastic process, we only need to prove that $\xi^{e}$ admits a representation. Define the function $T(t)=\left\|\xi_{t}^{e}\right\|$ and the set $\mathbb{J}=\left\{t \in \mathbb{R}_{+}: T(t)>\sup _{u>t} T(u)\right\}$ (with $\sup \varnothing=-\infty$ ). As $T$ is monotone, $\mathbb{J}$ is countable; let $\mathbb{C}$ be a countable subset of $\mathbb{R}_{+}$such that $T[\mathbb{C}]$ is dense in $T\left[\mathbb{R}_{+}\right]$. For each $t \in \mathbb{R}_{+}$either $t \in \mathbb{J}$ or there is a decreasing sequence $\left\langle t_{k}\right\rangle_{k \in \mathbb{N}}$ in $\mathbb{C}$ such that $\lim _{k} T\left(t_{k}\right)=T(t)$. Let $\left\langle t_{n}\right\rangle_{n \in \mathbb{N}}$ be an explicit enumeration of $\mathbb{D}=\mathbb{C} \cup \mathbb{J}$, choose $\bar{\xi}_{t_{n}}^{e} \in c a(\mathcal{F})$ such that $\bar{\xi}_{t_{n}}^{e} \mid \mathcal{A}_{t_{n}}=\xi_{t_{n}}^{e}$, fix $Q \in \mathbb{P}(\mathcal{F})$ and let $\bar{P}=Q+\sum_{n} 2^{-n} \bar{\xi}_{t_{n}}^{e}$ and $P=\|\bar{P}\|^{-1} \bar{P}$. Clearly, $P \in \mathbb{P}(\mathcal{F}), P \gg \bar{\xi}_{t_{n}}^{e}$ for each $n \in \mathbb{N}$. By construction, for each $t \in \mathbb{R}_{+}$and $k>0$ there is $t_{k} \in \mathbb{D}$ such that $t \leq t_{k}$ and $\left(\xi_{t}^{e}-\xi_{t_{k}}^{e}\right)(\Omega) \leq 2^{-k}$. Remark that $\left(\bar{\xi}_{t}^{e}-\bar{\xi}_{t_{k}}^{e}\right) \mid \sigma \mathcal{A}_{t} \in c a\left(\sigma \mathcal{A}_{t}\right)$ is the (unique) countably additive extension of $\xi_{t}^{e}-\xi_{t_{k}}^{e} \mid \mathcal{A}_{t}$ to $\sigma \mathcal{A}_{t}$ and is therefore positive. We conclude that $\bar{\xi}_{t}^{e}(F)=\lim _{k} \bar{\xi}_{t_{k}}^{e}(F)$ for each $F \in \sigma \mathcal{A}_{t}$. By Vitali Hahn Saks theorem and its corollaries [15, III.7.2-3], $\bar{\xi}_{t}^{e}\left|\sigma \mathcal{A}_{t} \ll P\right| \sigma \mathcal{A}_{t}$, i.e. $\xi^{e}$ is representable.

Uniformly countably additive supermartingales play a special role in the following section.

Proposition 2. Each $\xi \in \mathfrak{S}$ admits a unique decomposition $\xi=\xi^{u c}+\xi^{u p}$ where $\xi^{u c} \in \mathfrak{S}^{u c}$ and $\xi^{u p} \in \mathfrak{S}^{u p}$.

Proof. Let $\bar{x} \in \mathfrak{M}(\xi)$ and let $\bar{x}_{\overline{\mathcal{F}}}^{c}$ and $\bar{x}_{\overline{\mathcal{F}}}^{\perp}$ be the countably and purely finitely additive parts of $\bar{x} \mid \overline{\mathcal{F}}$, respectively. Define $\bar{x}^{\prime} \in \mathfrak{M}$ by letting

$$
\bar{x}^{\prime}(H)=\bar{x}_{\overline{\mathcal{F}}}^{c}\left(\bar{x}\left(H \mid \mathcal{I}_{\bar{x}_{\overline{\mathcal{F}}}}\right)\right) \quad H \subset \bar{\Omega}
$$

Then, by (2.3), $\bar{x}_{\overline{\mathcal{F}}}^{\prime}=\bar{x}_{\mathcal{F}}^{c}$ - so that $\bar{x}^{\prime} \in \mathfrak{M}^{u c}$. Letting $I_{n} \in \mathcal{I}_{\bar{x}_{\mathcal{F}}^{\perp}}$ be such that $\bar{x}_{\mathcal{F}}^{c}\left(I_{n}^{c}\right)<2^{-n}$

$$
\bar{x}^{\prime}(H)=\lim _{n} \bar{x}_{\overline{\mathcal{F}}}^{c}\left(I_{n} \bar{x}\left(H \mid \mathcal{I}_{\overline{\bar{x}} \frac{\perp}{\overline{\mathcal{F}}}}\right)\right)=\lim _{n} \bar{x}\left(I_{n} H\right) \leq \bar{x}(H) \quad H \subset \bar{\Omega}
$$

Clearly, $\bar{x}^{\prime \prime}=\bar{x}-\bar{x}^{\prime} \in \mathfrak{M}^{u p}$. Thus the set $\mathfrak{M}^{*}(\xi)=\left\{\bar{y} \in \mathfrak{M}^{u c}: \bar{y} \leq \bar{x}\right.$ for some $\left.\bar{x} \in \mathfrak{M}(\xi)\right\}$ is non empty and, we claim, it admits a maximal element with respect to the partial order $\geq_{\overline{\mathcal{F}}}$ defined by letting

\footnotetext{
${ }^{2}$ The property defined here was called the Kolmogoroff property by Bochner [8] p. 164]
} 
$\bar{y} \geq_{\overline{\mathcal{F}}} \bar{y}^{\prime}$ whenever $\bar{y}_{\overline{\mathcal{F}}} \geq \bar{y}_{\overline{\mathcal{F}}}^{\prime}$. In order to apply Zorn lemma, consider an increasing net $\left\langle\bar{y}^{\alpha}\right\rangle_{\alpha \in A}$ in $\mathfrak{M}^{*}(\xi)$ and let $\bar{x}^{\alpha} \in \mathfrak{M}(\xi)$ be such that $\bar{y}^{\alpha} \leq \bar{x}^{\alpha}$ for all $\alpha \in \mathbf{A}$. Define $\bar{x}^{\circ}(H)=\operatorname{LIM}_{\alpha \in \mathbf{A}} \bar{x}^{\alpha}(H), H \subset \bar{\Omega}-$ where LIM denotes the Banach limit functional introduced in [1]. By linearity, $\bar{x}^{\circ} \in \mathfrak{M}(\xi)$. The inequality $\bar{x}^{\circ}(H) \geq \liminf _{\alpha \in \mathbf{A}} \bar{x}^{\alpha}(H) \geq \liminf _{\alpha \in \mathbf{A}} \bar{y}^{\alpha}(H)$ which holds for any $H \subset \bar{\Omega}$ implies that $\bar{x}^{\circ} \geq_{\overline{\mathcal{F}}} \bar{y}^{\alpha}$ for all $\alpha \in \mathbf{A}$ i.e. that $\bar{x}^{\circ}$ is an upper bound for $\left\langle\bar{y}^{\alpha}\right\rangle_{\alpha \in A}$. Let $\bar{x}^{u c}$ be a maximal element of $\mathfrak{M}^{*}(\xi)$, let $\bar{x}^{*} \in \mathfrak{M}(\xi)$ be such that $\bar{x}^{u c} \leq \bar{x}^{*}$ and define $\bar{x}^{u p}=\bar{x}^{*}-\bar{x}^{u c} \in \mathfrak{M}$. Let $\xi^{u c}, \xi^{u p} \in \mathfrak{S}$ be uniquely defined by the condition $\bar{x}^{u c} \in \mathfrak{M}\left(\xi^{u c}\right), \bar{x}^{u p} \in \mathfrak{M}\left(\xi^{u p}\right), \xi_{\infty}^{u c}=\xi_{\infty}^{c}$ and $\xi_{\infty}^{u p}=\xi_{\infty}^{\perp}$. By construction, $\xi^{u c} \in \mathfrak{S}^{u c}$. Decompose $\bar{y} \in \mathfrak{M}\left(\xi^{u p}\right)$ as $\bar{y}^{\prime}+\bar{y}^{\prime \prime}$ where $\bar{y}^{\prime} \in \mathfrak{M}^{u c}$ and $\bar{y}^{\prime \prime} \in \mathfrak{M}^{u p}$, as in the first step of this proof. From $\bar{x}^{u c}+\bar{y}^{\prime} \leq \bar{x}^{u c}+\bar{y} \in \mathfrak{M}(\xi)$ and the fact that $\bar{x}^{u c}$ is $\geq_{\overline{\mathcal{F}}}$ maximal, we deduce $\bar{y}^{\prime}=0$ or, equivalently, $\xi^{u p} \in \mathfrak{S}^{u p}$. If $\xi=\kappa^{u c}+\kappa^{u p}$ were another such decomposition, and $k^{u p}$ and $k^{u c}$ the associated Doléans-Dade measures, then from $k^{u p} \leq x^{u c}+x^{u p}$ and Hahn Banach one may find $\bar{k}^{u p} \in \mathfrak{M}\left(\kappa^{u p}\right)$ such that $\bar{k}^{u p} \leq \bar{x}^{u c}+\bar{x}^{u p}$. However, since $\bar{k}^{u p} \perp \bar{x}^{u c}$, this implies $\bar{k}^{u p} \leq \bar{x}^{u p}$ while the converse is obtained mirrorwise. In other words $\kappa^{u p}$ and $\xi^{u p}$ induce the same Doléans-Dade measure; in addition, $\kappa_{\infty}^{u p}=\xi_{\infty}^{u p}=\xi_{\infty}^{\perp}$. The claim follows from Theorem $2($ iii $)$.

\section{InCREASING Processes}

Fix $P \in \mathbb{P}(\mathcal{F})$ and let $\mathfrak{A}(P)$ denote the set of processes $\left(A_{t}: t \in \mathbb{R}_{+}\right)$such that $A_{\infty} \in L^{1}(P)$ and $P\left(0=A_{0} \leq A_{t} \leq A_{u}\right)=1$ for each $0 \leq t \leq u<\infty$. Of course, if $A \in \mathfrak{A}(P)$ and $A^{\prime}$ is a modification of $A$ (i.e. $P\left(A_{t}^{\prime}=A_{t}\right)=1$ for all $\left.t \in \mathbb{R}_{+}\right)$then $A^{\prime} \in \mathfrak{A}(P)$. Put $\mathfrak{A}=\bigcup_{P \in \mathbb{P}(\mathcal{F})} \mathfrak{A}(P)$.

Lemma 3. Let $A \in \mathfrak{A}(P)$. Then there is $F \in \mathcal{F}$ with $P\left(F^{c}\right)=0$ and a modification $A^{\prime}$ of $A$ such that for each $t \leq u, 0=A_{0}^{\prime} \leq A_{t}^{\prime} \leq A_{u}^{\prime}$ on $F$. If in addition $P\left(A_{t}\right)=\lim _{n} P\left(A_{t+2^{-n}}\right)$ then $A^{\prime}$ and $F$ may be chosen to be right continuous at each $t \in \mathbb{R}_{+}$and for each $\omega \in F$.

Proof. As in the proof of Proposition 1, there exists a countable subset $\mathbb{D}$ of $\mathbb{R}_{+}$with the property that for each $t \in \mathbb{R}_{+}$and $\epsilon>0$ there is $d \in \mathbb{D}$ such that $d \geq t$ and $P\left(A_{t}\right)>P\left(A_{d}\right)-\epsilon$. Define $F=\bigcap_{\left\{d, d^{\prime} \in \mathbb{D}: d>d^{\prime}\right\}}\left\{A_{d} \geq\right.$ $\left.A_{d^{\prime}}\right\}$ : clearly, $P\left(F^{c}\right)=0$. Let $\mathbb{D}(t)=\{d \in \mathbb{D}: d \geq t\}$ and $A_{t}^{\prime}=\inf _{d \in \mathbb{D}(t)} A_{d}$. By definition of $\mathbb{D}, A_{t}^{\prime} \geq A_{t}$ but $P\left(A_{t}^{\prime}\right)=P\left(A_{t}\right)$ so that $P\left(A_{t}=A_{t}^{\prime}\right)=1$. If $A$ is right continuous in mean the same conclusion holds even if we replace $\mathbb{D}(t)$ with $\mathbb{D}^{+}(t)=\{d \in \mathbb{D}: d>t\}$. However $A^{\prime}$ is right continuous on $F$ since $\mathbb{D}^{+}(t)=\bigcap_{u>t} \mathbb{D}^{+}(u)$.

For $\left.\left.H=\left(F_{0} \times\{0\}\right) \cup \bigcup_{n=1}^{N}\left(F_{n} \times\right] t_{n}, u_{n}\right]\right) \in \overline{\mathcal{P}}$ and $A \in \mathfrak{A}(P)$ the integral $\int \mathbf{1}_{H} d A$ has an obvious definition, namely $\sum_{n=1}^{N} \mathbf{1}_{F_{n}}\left(A_{u_{n}}-A_{t_{n}}\right)$. In the following Theorem we obtain an extension of this integral together with a characterization of increasing processes in terms of their Doléans-Dade measure.

Theorem 3. Let $\bar{x} \in \mathfrak{M}$. The following are equivalent:

(i). There exists $P \in \mathbb{P}(\mathcal{F})$ and, given $P$, a unique (up to modification) $A \in \mathfrak{A}(P)$ such that

$$
\bar{x}(H)=P \int \mathbf{1}_{H} d A \quad H \in \overline{\mathcal{P}}
$$

(ii). $\quad \bar{x} \in \mathfrak{M}^{u c}$ and $\bar{x}(\{0\})=0$;

(iii). There exists $P \in \mathbb{P}(\mathcal{F})$ such that for each $h \in L^{1}(\bar{x})$ the equation

$$
\bar{x}(b h)=P\left(b I_{\bar{x}}(h)\right) \quad b \in \mathfrak{B}(\mathcal{F})
$$

admits a unique solution $I_{\bar{x}}(h) \in L^{1}(P)$ such that $I_{\bar{x}}\left(\mathbf{1}_{\{0\}}\right)=0$. 
$I_{\bar{x}}: L^{1}(\bar{x}) \rightarrow L^{1}(P)$ as defined in (5.2) is a positive, continuous, linear functional such that $\left\|I_{\bar{x}}\right\|=\|\bar{x}\|$ and that $\lim _{n} I_{\bar{x}}\left(h_{n}\right)=0$ whenever $\sup _{n}\left|h_{n}\right| \in L^{1}(\bar{x})$ and $\lim _{n} P^{*}\left(h_{n}^{*}>\eta\right)=0$ for each $\eta>0$ - where $h_{n}^{*} \equiv \sup _{t}\left|h_{n, t}\right|$ and $P^{*}$ is the outer measure generated by $P$.

Proof. Let us start remarking that one may easily identify $\overline{\mathcal{F}}$ with $\mathcal{F}$, as we shall now do. Under $(i)$, $\bar{x}(\{0\})=P \int \mathbf{1}_{\{0\}} d A=0$ and $\bar{x}(F)=\bar{x}(F \times] 0, \infty[)=P\left(\mathbf{1}_{F} A_{\infty}\right)$ for any $F \in \mathcal{F}$. Assume $(i i)$ and fix $P=(\|Q\|+\|\bar{x}\|)^{-1}(Q+\bar{x} \mid \overline{\mathcal{F}})$ for some $Q \in \mathbb{P}(\mathcal{F})$. By Theorem $\mathbb{1}$, for each $h \in L^{1}(\bar{x})$ we may define

$$
I_{\bar{x}}(h)=\bar{x}(h \mid \overline{\mathcal{F}}) \frac{d \bar{x} \mid \overline{\mathcal{F}}}{d P} \in L^{1}(P)
$$

By (2.2), $I_{\bar{x}}(h)$ is a solution to (5.2); moreover the operator $I_{\bar{x}}$ is positive, linear and has norm $\|\bar{x}\|$, by Theorem 1: $\bar{x}(\{0\})=0$ implies $I_{\bar{x}}\left(\mathbf{1}_{\{0\}}\right)=0, P$ a.s.. Any other solution $J(h) \in L^{1}(P)$ to (5.2) satisfies $P(b J(h))=P\left(b I_{\bar{x}}(h)\right)$ for all $b \in L^{\infty}(P)$ i.e. $P\left(J(h)=I_{\bar{x}}(h)\right)=1$. Assume $(i i i)$ and define $A_{t}=I_{\bar{x}}\left(\mathbf{1}_{\mathrm{j} 0, t]}\right)$, $A=\left(A_{t}: t \in \mathbb{R}_{+}\right)$and let $\left.\left.H=\left(F_{0} \times\{0\}\right) \cup \bigcup_{n=1}^{N}\left(F_{n} \times\right] t_{n}, u_{n}\right]\right) \in \overline{\mathcal{P}}$. Then $A \in \mathfrak{A}(P)$ and, up to a $P$ null set

$$
\begin{aligned}
I_{\bar{x}}\left(\mathbf{1}_{H}\right) & =\sum_{n=1}^{N} \bar{x}\left(\mathbf{1}_{\left.\left.F_{n} \times\right] t_{n}, u_{n}\right]} \mid \overline{\mathcal{F}}\right) \frac{d \bar{x} \mid \overline{\mathcal{F}}}{d P} \\
& =\sum_{n=1}^{N} \mathbf{1}_{F_{n}} \bar{x}\left(\mathbf{1}_{] t_{n}, u_{n}\right]} \mid \overline{\mathcal{F}}\right) \frac{d \bar{x} \mid \overline{\mathcal{F}}}{d P} \\
& =\sum_{n=1}^{N} \mathbf{1}_{F_{n}}\left(A_{u_{n}}-A_{t_{n}}\right) \\
& =\int \mathbf{1}_{H} d A
\end{aligned}
$$

But then (5.1) follows from (5.2). If $B \in \mathfrak{A}(P)$ also meets (5.1) then for $h=\mathbf{1}_{F \times] 0, t]}$ and $F \in \mathcal{F}$ we conclude that $P\left(F A_{t}\right)=P\left(F B_{t}\right)$ from which we deduce uniqueness. It is clear from (5.1) that $I_{\bar{x}}$ is linear, positive and that $\left\|I_{\bar{x}}\right\|=\|\bar{x}\|$.

If $\left\langle h_{n}\right\rangle_{n \in \mathbb{N}}$ is a sequence in $L^{1}(\bar{x})$ with the above properties then so is $\left\langle\left|h_{n}\right|\right\rangle_{n \in \mathbb{N}}$. Given that $I_{\bar{x}}$ is positive, it is enough to prove the claim for $h_{n} \geq 0$. Observe that $\bar{x}\left(h_{n} \geq \eta\right) \leq \bar{x}\left(h_{n}^{*} \geq \eta\right)$; moreover, $\bar{x} \mid \overline{\mathcal{F}} \ll P$ implies that, in restriction to $2^{\Omega} \otimes\left\{\varnothing, \mathbb{R}_{+}\right\}, \bar{x} \ll P^{*}$. But then, $h_{n}$ converges to 0 in $\bar{x}$ measure and, by [15, theorem III.3.6], in $L^{1}(\bar{x})$. Given (5.2) this is equivalent to $I_{\bar{x}}\left(h_{n}\right)$ converging to 0 in $L^{1}(P)$.

The equivalence of $(i)$ and $(i i)$ establishes a correspondence between $\mathfrak{A}$ and $\mathfrak{M}^{u c}$ which compares to the classical (and well known) characterization of increasing processes as measures given by Meyer [11, VI.65, p. 128] (see also [22, p. 6]). Meyer's result, which ultimately delivers the Doob Meyer decomposition, focuses however on countable additivity over $\mathcal{F} \otimes \mathcal{B}\left(\mathbb{R}_{+}\right)$; we rather require this property relatively to $\overline{\mathcal{F}}$. In Theorem 4 below we show that indeed this is enough to obtain a suitable version of Doob Meyer decomposition. Although there are connections between these two properties, it is noticeable that the latter is independent of the given filtration. On should also remark that we do not assume the existence of an underlying probability $P \in \mathbb{P}(\mathcal{F})$ but rather deduce it.

Each $\bar{x} \in \mathfrak{M}$ may be considered in restriction to special classes of functions such as the set $C$ of functions $f: \bar{\Omega} \rightarrow \mathbb{R}$ with continuous sample paths and bounded support (i.e. such that $f(t)=0$ for all $t$ larger than some $T$ ). Let $\mathcal{C}$ be the $\sigma$ algebra on $\bar{\Omega}$ generated by $C$. 
Lemma 4. Let $\bar{x} \in \mathfrak{M}^{c}$. There exist $\alpha^{c} \in c a(\mathcal{C})_{+}, P \in \mathbb{P}(\mathcal{F})$ and $A^{c} \in \mathfrak{A}(P)$ right continuous such that

$$
\bar{x}(f)=\alpha^{c}(f)=P \int f d A^{c} \quad f \in L(\bar{x}) \cap C
$$

Proof. In order to apply Daniell theorem, consider a sequence $\left\langle h_{n}\right\rangle_{n \in \mathbb{N}}$ in the vector lattice $L(\bar{x}) \cap C$ decreasing to 0 and fix $T$ such that $\bar{x}\left(\left|h_{1}-h_{1}^{T}\right|\right)<\epsilon$, where $h_{n}^{T}=h_{n} \mathbf{1}_{] 0, T]}$. Let $h_{n}^{T, *}=\sup _{t} h_{n}^{T}(t)$. A simple application of Dini's theorem for each $\omega \in \Omega$ guarantees that the sequence $\left\langle h_{n}^{T, *}\right\rangle_{n \in \mathbb{N}}$ converges to 0 pointwise; moreover, by continuity of the sample paths, $h_{n}^{T, *}$ is in fact $\mathcal{F}$ measurable. Thus Theorem 3 implies that $\lim _{n} \bar{x}\left(h_{n}\right) \leq$ $\epsilon+\lim _{n} \bar{x}\left(h_{n}^{T}\right)=\epsilon+\lim _{n} P\left(I_{\bar{x}}\left(h_{n}^{T}\right)\right)=\epsilon$. In other words, the restriction of $\bar{x}$ to $C$ is a Daniell integral and as such it admits the representation as the integral with respect to some $\alpha^{c} \in c a(\mathcal{C})$. Observe that $F \times] t, \infty\left[\in \mathcal{C}\right.$ for all $F \in \mathcal{F}$ and $t \in \mathbb{R}_{+}$. Fix $P \in \mathbb{P}(\mathcal{F})$ as in Theorem $3(i)$ and define $\alpha_{t}^{c} \in b a(\mathcal{F})$ as $\left.\left.\alpha^{c}(F \times] 0, t\right]\right)$ for each $F \in \mathcal{F}$. Since $\alpha_{t}^{c} \leq \bar{x}_{\overline{\mathcal{F}}} \ll P$, denote by $A_{t}^{c}$ the Radon Nikodym derivative of $\alpha_{t}^{c}$ with respect to $P$. We deduce that $\left.\left.\left.\left.P\left(\left(A_{u}^{c}-A_{t}^{c}\right) \mathbf{1}_{F}\right)=\alpha^{c}(F \times] t, u\right]\right)=\bar{x}(F \times] t, u\right]\right) \geq 0$, so that $A^{c} \in \mathfrak{A}(P)$, and that $\left.\left.\lim _{n} P\left(\mathbf{1}_{F}\left(A_{t+2^{-n}}^{c}-A_{t}^{c}\right)\right)=\lim \alpha^{c}(F \times] t, t+2^{-n}\right]\right)=0$ (by countable additivity) for each $F \in \mathcal{F}$ so that $A_{t}^{c}=\lim _{n} A_{t+2^{-n}}^{c}$ up to a $P$ null set. By Lemma 3, we obtain that $A^{c}$ admits a modification which is right continuous.

Theorem 4. Let $\xi \in \mathfrak{S}$. Then $\xi \in \mathfrak{S}^{u c}$ if and only if there exist $P \in \mathbb{P}(\mathcal{F}), M \in L^{1}(P)$ and $A^{p} \in \mathfrak{A}(P)$ which is adapted, right continuous in mean and such that

$$
\xi_{t}(F)=P\left(\mathbf{1}_{F}\left(M-A_{t}^{p}\right)\right) \quad t \in \mathbb{R}_{+} \text {and } F \in \mathcal{F}_{t}
$$

and that

$$
P\left(b \int h d A^{p}\right)=P \int M(b)_{-} h d A^{p} \quad b \in L^{\infty}(P), h \in \mathfrak{B}(\sigma \mathcal{P})
$$

where $M(b)=\left(P\left(b \mid \mathcal{F}_{t}\right): t \in \mathbb{R}_{+}\right)$.

Proof. We use the notation of Lemma 4 and the inclusion $\sigma \mathcal{P} \subset \mathcal{C}$. Let $d=\left\{t_{1} \leq t_{2} \leq \ldots \leq t_{N}\right\}$ be a finite sequence in $\mathbb{R}_{+}$and define

$$
\mathcal{P}^{d}(f)=\sum_{n=1}^{N-1} P\left(f_{t_{n}} \mid \mathcal{F}_{t_{n}}\right) \mathbf{1}_{\left.t_{n}, t_{n+1}\right]} \quad \text { and } \quad \bar{x}^{d}(f)=\bar{x}\left(\mathcal{P}^{d}(f)\right) \quad f: \mathbb{R}_{+} \rightarrow L^{1}(P)
$$

Denote by $\alpha^{d}$ the restriction of $\bar{x}^{d}$ to $\mathcal{F} \otimes 2^{\mathbb{R}_{+}}$. On the one hand it is easily seen that $\bar{x}_{\overline{\mathcal{F}}}^{d} \ll P$ so that, as in the proof of Theorem 31, we can associate to $\bar{x}^{d}$ a process $A^{d} \in \mathfrak{A}(P)$, by letting $A_{t}^{d} d P=\bar{x}^{d}\left(\mathbf{1}_{] 0, t]} \mid \overline{\mathcal{F}}\right) d \bar{x}_{\overline{\mathcal{F}}}^{d}$. On the other hand, (5.3) implies $P \int f d A^{d}=\bar{x}\left(\mathcal{P}^{d}(f)\right)=\alpha^{c}\left(\mathcal{P}^{d}(f)\right)$. Consider the case in which $f=b h$ where $b \in L^{\infty}(P)$ and $h$ is bounded, adapted and left continuous. Let $d_{n}=\left\{k 2^{-n}: k=0, \ldots, 2^{2 n}\right\}$ and observe that, by [11, VI.2, p. 67], there exists a $P$ null set $F \in \mathcal{F}$ outside of which $\lim _{n} \mathcal{P}^{d_{n}}(b)_{t}=M(b)_{t-}$ and $\lim _{n} \mathcal{P}^{d_{n}}(h)_{t}=h_{t}$ for each $t \in \mathbb{R}_{+}$(as $h$ is left continuous and adapted). Given that $\alpha^{c}$ is countably additive in restriction to $\mathcal{C}$, we conclude

$$
\lim _{n} P\left(b \int h d A^{d_{n}}\right)=\lim _{n} \alpha^{c}\left(\mathcal{P}^{d_{n}}(b h)\right)=\lim _{n} \alpha^{c}\left(\mathcal{P}^{d_{n}}(b) \mathcal{P}^{d_{n}}(h)\right)=\alpha^{c}\left(M(b)_{-} h\right)
$$

Define then $\alpha^{p} \in b a\left(\mathcal{F} \otimes 2^{\mathbb{R}_{+}}\right)$implicitly as $\alpha^{p}(H)=\alpha^{c}\left(M\left(\mathbf{1}_{H}\right)_{-}\right)$. Then from (5.7) we deduce that $\left\langle\alpha^{d_{n}}\right\rangle_{n \in \mathbb{N}}$ converges to $\alpha^{p}$ and, by [15, III.7.3, p. 159], that $\alpha_{\overline{\mathcal{F}}}^{p} \ll P$. Let $A^{p} \in \mathfrak{A}(P)$ be the increasing process associated to $\alpha^{p}$. Thus for every bounded, adapted and left continuous process $h$ and every $b \in L^{\infty}(P)$ we have

$$
P\left(b \int h d A^{p}\right)=\bar{x}\left(M(b)_{-} h\right)=\alpha^{c}\left(M(b)_{-} h\right)=P \int M(b)_{-} h d A^{p}
$$


which delivers (5.4) if we only let $M=d \xi_{\infty} / d P+A_{\infty}^{p}, b=\mathbf{1}_{F}$ with $F \in \mathcal{F}_{t}$ and $h=\mathbf{1}_{] t, \infty[}$. In addition, if $F \in \mathcal{F}, s \leq t$ and $h_{F, t}=\mathbf{1}_{F}-P\left(\mathbf{1}_{F} \mid \mathcal{F}_{t}\right)$, then $M\left(h_{F, t}\right)_{s-}=0$ so that

$$
P\left(h_{F, t} A_{t}^{p}\right)=\alpha^{p}\left(h_{F, t} \mathbf{1}_{[0, t]}\right)=P \int_{0}^{t} M\left(h_{F, t}\right)_{-} d A^{p}=0
$$

Therefore, replacing $A_{t}^{p}$ with $P\left(A_{t}^{p} \mid \mathcal{F}_{t}\right)$, we may assume that $A^{p} \in \mathfrak{A}(P)$ is adapted. Eventually, letting $h_{n}=\mathbf{1}_{\left.] t, t+2^{-n}\right]}$ we conclude that $0=\lim _{n} \alpha^{c}\left(h_{n}\right)=\lim _{n} P\left(A_{t+2^{-n}}^{p}-A_{t}^{p}\right)$ and, thus, that $A^{p}$ is right continuous in mean. That (5.4) implies $\xi \in \mathfrak{S}^{u c}$ is obvious.

With a complete filtration Theorem 4 implies that $A^{p}$ may be chosen to be adapted and right continuous.

We want to emphasize that the existence of the decomposition (5.4) does not depend on the underlying filtration.

Corollary 3. Let $\xi \in \mathfrak{S}$ and let $\mathbb{D} \subset \mathbb{R}_{+}$be such that $\xi_{t}=\sup _{d \in \mathbb{D}(t)} \xi_{d} \mid \mathcal{A}_{t}$ where $\mathbb{D}(t)=\{d \in \mathbb{D}: d \geq t\}$. Then, $\xi$ admits a Doob Meyer decomposition if and only if $\xi^{\mathbb{D}}=\left(\xi_{d}: d \in \mathbb{D}\right)$ does.

Proof. Given that, by Theorem 4, the Doob Meyer decomposition is equivalent to $\xi \in \mathfrak{S}^{u c}$, the direct implication is obvious. As for the converse, choose $\bar{x}^{\mathbb{D}} \in \mathfrak{M}\left(\xi^{\mathbb{D}}\right)$ to be countably additive in restriction to $\mathcal{F} \otimes\{\varnothing, \mathbb{D}\}$. If $t \in \mathbb{R}_{+}$and $F \in \mathcal{A}_{t}$ then

$$
\left|\xi_{t}\right|(F) \leq \sup _{d \in \mathbb{D}(t)}\left|\xi_{d}\right|(F) \leq\left|\xi_{\infty}\right|(F)+\bar{x}^{\mathbb{D}}(F \times \mathbb{D}) \equiv \lambda(F)
$$

The claim then follows from Corollary 1 .

Corollary 3 makes clear that decomposition (5.4) is a property that involes any subset $\mathbb{D}$ which is dense for the range of $\xi$ and we know from the proof of Proposition 1 that this may be taken to be countable. The class $D$ property may thus be replaced by a corresponding property, the class $D_{\sigma}$, in which the stopping times are restricted to have countable range, see [9].

\section{The Bichteler Dellacherie Theorem without Probability}

Let $f: \bar{\Omega} \rightarrow \mathbb{R}$ be adapted to the filtration, define $f^{*}=\sup _{t \in \mathbb{R}_{+}}\left|f_{t}\right|$ and let $\mathcal{F}$ be such that $f^{*}$ is $\mathcal{F}$ measurable. The starting point of this section are the sets

$$
\mathcal{K}=\left\{\int h d f: h \text { is } \mathcal{P} \text { simple, }|h| \leq 1\right\} \quad \text { and } \quad \mathcal{C}=\mathcal{K}-\mathfrak{B}(\mathcal{F})_{+}
$$

Bichteler and Dellacherie start from the assumption that $\mathcal{K}$ is bounded in $L^{0}(P)$ for some given $P \in \mathbb{P}(\mathcal{F})$ and that $f$ is right continuous with left limits outside some $P$ null set. These two properties are then shown to imply that for given $\eta>0$ there exists $\delta>0$ such that $d \mathbf{1}_{F} \notin \mathcal{C} \mathbf{1}_{F}$ for all $F \in \mathcal{F}$ such that $P(F)>\eta$. We take inspiration from this separating condition to define a concept of boundedness suitable for our setting. To this end we denote by $\mathcal{U}$ a collection of subets of $\Omega$ with the following properties:

Assumption 1. There exists $\lambda_{0}>0$ such that

$$
\left\{\lambda \mathbf{1}_{U}: \lambda \geq \lambda_{0}\right\} \cap \mathcal{C} \mathbf{1}_{U}=\varnothing \quad U \in \mathcal{U}
$$

Moreover, $U, V \in \mathcal{U}$ imply $U \subset\left\{f^{*}<n\right\}$ for some $n$ and $U \cup V \in \mathcal{U}$. 
A violation of (6.2) indicates that the set $\mathcal{K}$ is unbounded relatively to some $U \in \mathcal{U}$. Both sets in (6.2) are convex subsets of $\mathfrak{B}\left(2^{U}\right)$ and $\mathbf{1}_{U} \mathcal{C}$ contains $-\mathbf{1}_{U}$ as an internal point. By ordinary properties of the support functional [15, lemma V.I.8(f), p. 411], the Hahn Banach theorem and [15, lemma V.II.7, p. 417] we conclude that for each $U \in \mathcal{U}$ there is $\hat{m}_{U} \in b a\left(2^{U}\right)$ such that $\sup _{x \in \mathbf{1}_{U} \mathcal{C}} \hat{m}_{U}(x) \leq 1=\lambda_{0} \hat{m}_{U}(U)$. The inclusion $-\mathfrak{B}\left(2^{U}\right)_{+} \subset \mathbf{1}_{U} \mathcal{C}$ implies that $\hat{m}_{U} \geq 0$. By defining $m_{U} \in b a_{+}$implicitly by

$$
m_{U}(F)=\frac{\hat{m}_{U}(F \cap U)}{\hat{m}_{U}(U)} \quad F \subset \Omega
$$

we have completed the proof of the following:

Lemma 5. Let $f: \bar{\Omega} \rightarrow \mathbb{R}_{+}$satisfy Assumption 1 and define the set

$$
\mathcal{M}=\left\{m \in b a_{+}:\|m\|=1, \sup _{x \in \mathcal{C}} m(x) \leq \lambda_{0}\right\}
$$

For each $U \in \mathcal{U}$ there exists $m_{U} \in \mathcal{M}$ such that $m_{U}(U)=1$.

Fix now $m \in \mathcal{M}$ (so that $\left.f^{*} \in L^{1}(m)\right)$ and let $\xi^{e}$ and $\xi^{p}$ be the components of the finitely additive supermartingale $\left(m \mid \mathcal{F}_{t}: t \in \mathbb{R}_{+}\right)$as of (4.2). Set also $\mathcal{I}_{t}=\mathcal{I}_{\xi_{t}^{p}}$ (see (2.1)), let $(P, X)$ be a representation for $\xi^{e}$ and observe that $-\xi^{p} \in \mathfrak{S}$ and that $\mathfrak{M}\left(\xi^{e}\right)=\mathfrak{M}\left(-\xi^{p}\right)$. Fix an extension $\bar{\xi}_{\infty} \in b a(\mathcal{F})$ of $\xi_{\infty}$ to $\mathcal{F}$ and $\bar{x} \in \mathfrak{M}\left(\xi^{e}\right)$ and define

$$
\bar{\xi}_{t}^{p}(F)=\bar{\xi}_{\infty}^{p}(F)-\bar{x}(F \times] t, \infty[) \quad F \in \mathcal{F}
$$

The collection $\left(\bar{\xi}_{t}^{p}: t \in \mathbb{R}_{+}\right)$is then increasing with $t$. For each $b \in \mathfrak{B}(\mathcal{F})$ and $u \geq t, F \in \mathcal{I}_{t}$ implies $\xi_{\infty}^{p}\left(b \mathbf{1}_{F}\right)=\bar{x}\left(b \mathbf{1}_{F \times] t, \infty[}\right)$ and thus

$$
\bar{\xi}_{u}^{p}\left(b \mathbf{1}_{F}\right)=\xi_{\infty}^{p}\left(b \mathbf{1}_{F}\right)-\bar{x}\left(b \mathbf{1}_{F \times] u, \infty[}\right)=\bar{x}\left(b \mathbf{1}_{F \times] t, u]}\right)
$$

Let now $d=\left\{t_{1} \leq \ldots \leq t_{N}\right\}$

$$
\left.\left.\mathcal{P}^{d}=\left\{F_{0} \times\{0\} \cup \bigcup_{n=1}^{N-1} F_{n} \times\right] t_{n}, t_{n+1}\right]: F_{0} \in \mathcal{F}_{0}, F_{n} \in \mathcal{F}_{t_{n}}, 1 \leq n \leq N-1\right\}
$$

and choose $F_{n} \in \mathcal{I}_{t_{n}} 1 \leq n<N$ and set

$$
\left.\left.F^{d}=\bigcup_{n=1}^{N-1} F_{n} \times\right] t_{n}, t_{n+1}\right] \quad \text { and } \quad f^{d}=f_{0} \mathbf{1}_{\{0\}}+\sum_{n=1}^{N-1} f_{t_{n+1}} \mathbf{1}_{] t_{n}, t_{n+1}\right]}
$$

By (6.4)

$$
\begin{aligned}
\sum_{n=1}^{N-1} \xi_{t_{n+1}}^{p}\left(\left(f_{t_{n+1}}-f_{t_{n}}\right) \mathbf{1}_{F_{n}}\right) & =\sum_{n=1}^{N-1} \bar{x}\left(\left(f_{t_{n+1}}-f_{t_{n}}\right) \mathbf{1}_{\left.\left.F_{n} \times\right] t_{n}, t_{n+1}\right]}\right) \\
& =\bar{x}\left(f^{d} \mathbf{1}_{F^{d}}\right)-x\left(\sum_{n=1}^{N-1} f_{t_{n}} \mathbf{1}_{\left.\left.F_{n} \times\right] t_{n}, t_{n+1}\right]}\right) \\
& =\bar{x}\left(f^{d} \mathbf{1}_{F^{d}}\right)+P \sum_{n=1}^{N-1} f_{t_{n}} \mathbf{1}_{F_{n}}\left(X_{t_{n+1}}-X_{t_{n}}\right)
\end{aligned}
$$


i.e.

$$
\begin{aligned}
m\left(\int \mathbf{1}_{F^{d}} d f\right) & =\sum_{n=1}^{N-1} m\left(\left(f_{t_{n+1}}-f_{t_{n}}\right) \mathbf{1}_{F_{n}}\right) \\
& =\sum_{n=1}^{N-1}\left(\xi_{t_{n+1}}^{p}+\xi_{t_{n+1}}^{e}\right)\left(\left(f_{t_{n+1}}-f_{t_{n}}\right) \mathbf{1}_{F_{n}}\right) \\
& =\bar{x}\left(f^{d} \mathbf{1}_{F^{d}}\right)+P \sum_{n=1}^{N-1} \mathbf{1}_{F_{n}}\left(f_{t_{n+1}} X_{t_{n+1}}-f_{t_{n}} X_{t_{n}}\right)
\end{aligned}
$$

Assume that $H=H_{0} \mathbf{1}_{\{0\}}+\bigcup_{n=1}^{N-1} H_{n} \mathbf{1}_{\left.] t_{n}, t_{n+1}\right]} \in \mathcal{P}^{d}$. Then by (3.5)

$$
\bar{x}(H)=P\left\{\mathbf{1}_{H_{0}}\left(X_{0}-X_{\infty}\right)+\sum_{n=1}^{N-1} \mathbf{1}_{H_{n}}\left(X_{t_{n}}-X_{t_{n+1}}\right)\right\}
$$

i.e. $\bar{x} \mid \mathcal{P}^{d}$ is countably additive. Replacing $F^{d}$ with a sequence $\left\langle F^{d, k}\right\rangle_{k \in \mathbb{N}}$ such that $\lim _{k} P\left(\bigcap_{n=1}^{N-1} F_{n-1}^{k}\right)=1$ we thus deduce then from (6.7)

$$
\lim _{k} m\left(\int \mathbf{1}_{F^{d, k}} d f\right)=\bar{x}\left(f^{d}\right)+P\left(f_{\infty} X_{\infty}-f_{0} X_{0}\right)
$$

Replace $f$ with $\int \mathbf{1}_{F \times] t, u]} d f$ where $t \leq u$ and $F \in \mathcal{F}_{t}$ and choose $d$ such that $\left.\left.F \times\right] t, u\right] \in \mathcal{P}^{d}$. We also deduce

$$
\lim _{k} m\left(\mathbf{1}_{F} \int_{t}^{u} \mathbf{1}_{F^{d, k}} d f\right)=P\left(F\left(f_{u} X_{u}-f_{t} X_{t}\right)\right)+\bar{x}\left(f^{d} \mathbf{1}_{F \times] t, u]}\right)
$$

Theorem 5. Let $f \in \mathbb{R}^{\bar{\Omega}}$ satisfy Assumption 1 , Then there exists $P \in \mathbb{P}(\mathcal{F})$ and a $P$ positive supermartingale $X$ such that $X f$ is a $P$ quasimartingale. If there is $Q \in \mathbb{P}(\mathcal{F})$ and $\eta>0$ such that $Q\left(f^{*}<\infty\right)=1$ and that $F \in \mathcal{F}$ and $Q\left(F \cap\left\{f^{*}<k\right\}\right) \geq \eta$ imply $F \cap\left\{f^{*}<k\right\} \in \mathcal{U}$ then for any $\delta>\eta$ the pair $(P, X)$ above can be chosen such that $P\left(X_{\infty}=0\right)<\delta$.

Proof. By Lemma 5 for fixed $n>n_{0}$ there is $m \in \mathcal{M}$ such that $m\left(f^{*}>n\right)=0$ so that $m\left(f^{*}\right)<\infty$. By (6.4),

$$
\left|\bar{x}\left(f^{d}\right)\right| \leq \sum_{n=1}^{N-1}\left(\bar{\xi}_{t_{n+1}}^{p}-\bar{\xi}_{t_{n}}^{p}\right)\left(\sup _{1<j \leq N}\left|f_{t_{j}}\right|\right)=\bar{\xi}_{\infty}^{p}\left(\sup _{1<j \leq N}\left|f_{t_{j}}\right|\right) \leq m\left(f^{*}\right)
$$

Let $h_{n}^{d}$ be the sign of $P\left(f_{t_{n+1}} X_{t_{n+1}} \mid \mathcal{F}_{t_{n}}\right)-f_{t_{n}} X_{t_{n}}$ and $h^{d}=\sum_{n} h_{n}^{d} \mathbf{1}_{\left.t_{n}, t_{n+1}\right]}$. By (6.8)

$$
\begin{aligned}
P \sum_{n}\left|P\left(f_{t_{n+1}} X_{t_{n+1}} \mid \mathcal{F}_{t_{n}}\right)-f_{t_{n}} X_{t_{n}}\right| & =P \sum_{n} h_{n}^{d}\left(f_{t_{n+1}} X_{t_{n+1}}-f_{t_{n}} X_{t_{n}}\right) \\
& =\lim _{k} m\left(\int F^{d, k} h^{d} d f\right)-\bar{x}\left(f^{d} h^{d}\right) \\
& \leq \sup _{k \in \mathcal{K}} m(k)+m\left(f^{*}\right)
\end{aligned}
$$

Since such bound is uniform in $d$ this proves the first claim.

Let $Q \in \mathbb{P}(\mathcal{F})$ and $\eta$ be as in the claim. In search of a contradiction, assume that for some $\delta>\eta$ and all representations $(P, X)$ of $\xi^{e}$ we have $P\left(X_{\infty}=0\right)>\delta$. Fix $\epsilon=\delta-\eta$, define $P^{\epsilon}=(1-\epsilon) Q+\epsilon P$ and let $\left(P^{\epsilon}, X^{\epsilon}\right)$ be the corresponding representation. Given that $P^{\epsilon}$ and $\xi_{\infty}^{p}$ are orthogonal and that $\mathcal{F}$ is a $\sigma$ algebra, there is an $\mathcal{F}$ measurable subset $F$ of $\left\{X_{\infty}^{\epsilon}=0\right\}$ such that $\xi_{\infty}^{p}(F)=m(F)=0$ and $P^{\epsilon}(F)>\delta$ so that $Q\left(F\left\{f^{*}<k\right\}\right) \geq \frac{\delta-\epsilon}{1-\epsilon}=\frac{\eta}{1-\epsilon}$ for some integer $k$. We conclude that for each $m \in \mathcal{M}$ there exists 
$h_{m} \in \mathfrak{B}(\mathcal{F})$ such that $0 \leq h_{m} \leq 1, Q\left(h_{m}\right) \geq \frac{\eta}{1-\epsilon},\left\{h_{m}>0\right\} \in \mathcal{U}$ and $m\left(h_{m}\right)=0$. Denote by $H$ the corresponding collection. Then

$$
\sup _{m \in \mathcal{M}} \inf _{h \in H} m(h)=0
$$

Endow $\mathfrak{B}(\mathcal{F})$ with the norm topology and $b a(\mathcal{F})$ with the weak* topology. One easily remarks that $H$ and $\mathcal{M}$ are convex sets (as $\mathcal{U}$ is closed with respect to unions) and that $\mathcal{M}$, being a closed subset of the unit sphere of $b a(\mathcal{F})$, is compact; moreover, the function $(m, h) \rightarrow m(h)$ is bilinear and separately continuous. Sion's [24, corollary 3.3, p. 174] version of the minimax theorem therefore applies, yielding the conclusion

$$
\inf _{h \in H} \sup _{m \in \mathcal{M}} m(h)=0
$$

There is then a sequence $\left\langle h_{n}\right\rangle_{n \in \mathbb{N}}$ in $H$ such that $\sup _{m \in \mathcal{M}} m\left(h_{n}\right)<2^{-n}$. Given that $H$ is convex we may equivalently replace $h_{n}$ by a convex combination $\sum_{j=0}^{J} \alpha_{j} h_{n+j}$. As a consequence of Komlos lemma [19, theorem 1, p. 218] (but see also [23, theorem 6, p. 184]) there is no loss of generality in assuming that the sequence $\left\langle h_{n}\right\rangle_{n \in \mathbb{N}}$ converges $Q$ a.s. to some $h^{\prime}$. By Egoroff theorem, we can choose $F \in \mathcal{F}$ such that $h_{n}$ converges uniformly to $h^{\prime}$ on $F$ and that, letting $h=h^{\prime} \mathbf{1}_{F}$,

$$
Q(h)=\lim _{n} Q\left(h_{n} \mathbf{1}_{F}\right) \geq \lim _{n} Q\left(h_{n}\right)-Q\left(F^{c}\right) \geq \frac{\eta}{1-\epsilon / 2}>\eta
$$

while

$$
\sup _{m \in \mathcal{M}} m(h) \leq \lim _{n} \sup _{m \in \mathcal{M}} m\left(h_{n}\right)=0
$$

Both inequalities remain true if we replace $h$ by $U=\left\{h>a ; f^{*}<1 / a\right\}$ for $a$ sufficiently small. Then $U \in \mathcal{U}$ but $m(U)=0$ for all $m \in \mathcal{M}$ so that Lemma 5 fails, a contradiction.

\section{REFERENCES}

[1] R. P. Agnew, A. P. Morse (1938), Extensions of Linear Functionals, with Applications to Limits, Integrals, Measures, and Densities, Ann. Math., 39, 20-30.

[2] T. Armstrong (1983), Finitely Additive F-processes, Trans. Amer. Math. Soc. 279, 271-295.

[3] T. Armstrong (1985), Finitely Additive Supermartingales and Differences of Martingales and Adapted Increasing Processes, Proc. Amer. Math. Soc. 95, 619-625 .

[4] P. Berti, P. Rigo (2004), Convergence in Distribution of Non-measurable Random Elements, Ann. Prob. 32, 365-379.

[5] K. P. S. Bhaskara Rao, M. Bhaskara Rao (1983), Theory of Charges, Academic Press, London.

[6] S. Bochner (1947), Stochastic Processes, Ann. Math. 48, 1014-1061.

[7] S. Bochner (1950), Partial Ordering in the Theory of Stochastic Processes, Proc. Nat. Acad. Sc. 36, 439-443.

[8] S. Bochner (1955), Partial Ordering in the Theory of Martingales, Ann. Math. 62, 162-169.

[9] G. Cassese (2007), Decomposition of Supermartingales Indexed by a Linearly Ordered Set, Stat. Prob. Lett., 77, 795-802.

[10] G. Cassese (2008), Asset Pricing with No Exogenous Probability Measure, Math. Fin., 18, 23-54.

[11] C. Dellacherie, P. A. Meyer (1982), Probabilities and Potential B, North-Holland, Amsterdam.

[12] C. Doléans-Dade (1968), Existence du Processus Croissant Naturel Associé à un Potentiel de la Classe (D), Z. Wahrsch. Verw. Geb. 9, 309-314.

[13] L. Dubins (1975), Finitely Additive ConditionalProbabilities, Conglomerability and Disintegrations, Ann. Prob. 3, 79-99.

[14] R. M. Dudley (1990), Non Metric Compact Spaces and non Measurable Processes, Ann. Prob. 108, $1001-05$.

[15] N. Dunford, J. Schwartz (1988), Linear Operators, Wiley, New York.

[16] H. Föllmer (1973), On the Representation of Semimartingales, Ann. Prob., 5, 580-589.

[17] J. B. Kadane, M. J. Schervish, T. Seidenfeld (1996), Reasoning to a Foregone Conclusion, J. Amer. Stat. Ass. 91 , 1228-1235.

[18] I. Karatzas, G. Zitkovic (2003), Optimal Consumption from Investment and Random Endowment in Incomplete Semimartingale Markets, Ann. Prob. 31, 1821-1858. 
[19] J. Komlos (1967), A Generalization of a Problem of Steinhaus, Acta Math. Hung. 18, 217-229.

[20] J. F. Mertens (1972), Processus Stochastiques Généraux et Surmartingales, Z. Wahrsch. Verw. Geb. 22, 45-68.

[21] M. Metivier, J. P. Pellaumail (1975), On Doléans-Föllmer Measure for Quasi-Martingales, Ill. J. Math. 19, $491-504$.

[22] P. A. Meyer (1976), Un Cours sur les Integrales Stochastiques, Sém. Prob. 10, 245-400

[23] M. Schwartz (1986), New Proofs of a Theorem of Komlos, Acta Math. Hung. 45, 181-185.

[24] M. Sion (1958), On General Minimax Theorems, Pacific J. Math. 8, 171-176.

[25] Y. A. Yan (1980), Caractérization d'une Classe d'Ensembles Convexes de $L^{1}$ ou $H^{1}$, Sem. Prob. 12, $220-222$.

[26] A. W van der Vaart, J. A. Wellner (1996), Weak Convergence and EMpirical Processes, Springer-Verlag, New York

[27] K. Yosida (1952), E. Hewitt, Finitely Additive Measures, Trans. Amer. Math. Soc. 72, 46-66.

Università del Salento And University of Lugano

E-mail address: g.cassese@economia.unile.it

Current address: Dipartimento di Scienze Economiche e Matematico-Statistiche, Ecotekne, via per Monteroni, 73100 Lecce 